\title{
Using Ultrasonic Spectrometry to Estimate the Stability of a Dental Implant Phantom
}

\author{
Hamed Hamid Muhammed, Satya V. V. N. Kothapalli \\ School of Technology and Health STH, Royal Institute of Technology KTH Alfred Nobels Alle 10, \\ Stockholm, Sweden \\ Email: hamed.muhammed@sth.kth.se, satya.kothapalli@sth.kth.se
}

Received 2013

\begin{abstract}
A challenging problem in dental implant surgery is to evaluate the stability of the implant. In this simulation study, an experimental phantom is used to represent a jawbone with a dental implant. It is made of a little pool filled with soft-tissue equivalent material and a disc of fresh Oakwood with a metal screw. Varying levels of contact between screw and wood are simulated by screwing in or out the screw. Initially, the screw is screwed in and fixed firmly in wood. Thereafter, the screw is screwed out, a half turn each time, to increase the gap gradually between wood and screw. Pulse-echo ultrasound is used and the power spectra of the received echo-signals are computed. These spectra are normalized then analyzed by using the partial least squares method to estimate the corresponding implant stiffness grade in terms of number of turns when beginning from the initial tight-screw state then screwing out the screw. A coefficient of determination $\mathrm{R}^{2}$ of $96.4 \%$ and a mean absolute error of \pm 0.23 turns are achieved when comparing real and estimated values of stiffness grades, indicating the efficiency of this approach.
\end{abstract}

Keywords: Dental Implant Stability; Partial Least Squares; Ultrasonic Spectrometry; Spectral Analysis

\section{Introduction}

Nowadays, dental implant surgeries are common among female, male, young and elderly patients. In general, these surgeries are performed in four different phases. The most critical phase is the second one, called osseointegration [1], where the integration of implant into living jawbone occurs. Depending on many factors, including age, gender, bone tissue density and pathological condition of the patient, the osseointegration process may take a longer or a shorter time period.

Therefore, it is important to have a non-destructive, risk-free and mobile clinical routine to measure the fixation of the implant. Fitting and Adler [2] suggested the use of ultrasonic spectral analysis for non-destructive testing. By using this technique, which can be optimized to fulfill the requirements mentioned above, it sounds promising to test the hypothesis that ultrasonic spectral measurements can be performed to evaluate the biomechanical stability or the stiffness of the bone-implant interface which is proportional to the osseointegration grade.

Recently, Shiu-Fen Lin et al. [3] as well as Pan and Ying [4] proposed and used resonance frequency analysis (RFA) to evaluate the grade of osseointegration. They found that the resonance frequency of the bone-implant interface or structure was proportional to implant mechanical stability. The frequencies considered were in the range of $60-120 \mathrm{~Hz}$ to be able to study the vibration of the whole implant imbedded into Bakelite (a gypsum model). The results presented were rather binary, only showing if the grade of stiffness was high or low.

Valderrama et al. [5] showed that the recently introduced magnetic RFA device could give comparable results as the original electronic variant. However, Pattijnet $a l$. [6] showed that the energy of the signal measured by the RFA technique was angle and displacement dependent and could change considerably when performing the measurements at different parts of the implants and from different directions.

De Almeida et al. [7] introduced a new approach, called quantitive ultrasound (QUS), where a transmission ultrasound technique was used to inspect a phantom made of a metal threaded piece (representing the implant) imbedded into a metal block (corresponding to the bone). A $1 \mathrm{MHz}$ central frequency ultrasonic transducer was used in this study. The results showed that the mean value of detected time signal was proportional to the stiffness of the structure of this phantom.

Mathieu et al. [8] performed an ex vivo study and showed that the QUS technique could be used to compute a quantitative parameter which was significantly 
sensitive to the amount of bone (rabbit femur was used) in contact with a cylindrical titanium dental implant. The transducer used in this study had a central frequency of $10 \mathrm{MHz}$ to allow for distinguishing different echoes originating from different implant interfaces. Furthermore, a long-enough signal duration (25 times longer that used by de Almeida et al. [7]) was used to retrieve more information from the implant and compute the indicator.

The new approach, proposed by this work, aims at evaluating the stability of the dental implant screw in bone and soft tissues. For this purpose, a proper phantom is designed and used. Ultrasonic spectral analysis is utilized to detect differences or changes in the shape of the curve of the power spectrum of the ultrasonic echo-signals reflected from the phantom representing an implant imbedded into a jawbone. An automatic statistical-modeling method is developed and utilized to estimate (based on analyzing the shape of ultrasonic power spectra) the contact and the stiffness grade between the implant and the jawbone.

However, in statistical modeling, two common problems are usually encountered: 1) Large number of variables and few observations. 2) Explanatory and dependent variables are collinear. These two problems can be solved by, at first, preprocessing and normalizing the data in an efficient way, then using a suitable Partial Least Squares (PLS) algorithm to achieve the desired results.

\section{Materials and Methods}

\subsection{Phantom and Experimental Setup}

A disc of fresh Oakwood was used to simulate a jawbone. The speed of sound in Oakwood is about $3800 \mathrm{~m} / \mathrm{s}$ [9], which is close to the speed of sound in bone tissue, which is about $3750 \pm 250 \mathrm{~m} / \mathrm{s}$. The spongy microstructure of wood tissue is also similar to bone tissue making it efficient to produce and use wood-based bone-implants [10]. A metal screw was screwed into this wooden disc to simulate an implant, as shown in Figure 1(a). This disc was immersed into a pool filled with water and a soft-tissue equivalent material; a black-colored mixture composed of $4 \%$ graphite, 3\% agar and 93\% water [11]. An ultrasonic transducer was mounted at $21 \mathrm{~mm}$ distance from the edge of the wooden disc as shown in Figure 1(b). The central or fundamental frequency of this singlecrystal piezoelectric ultrasonic transducer was $2 \mathrm{MHz}$, and the frequency band was ranging from $1.8 \mathrm{MHz}$ to 2.2 $\mathrm{MHz}$

A pulse generator with an amplifier was used to excite the transducer to make it emit an ultrasonic pulse, then receive the echo signal and transfer it through the amplifier to an oscilloscope. A power spectrum of the received signal was generated by the oscilloscope by using the fast Fourier transform (FFT). This spectrum was transferred to a personal computer (PC), where it was analyzed.

\subsection{Dataset}

A set of 30 power spectra was acquired by the pulse-echo ultrasound system described previously. Each power spectrum corresponded to a certain contact level and a certain stiffness grade between the metal screw and the wooden disc. The contact level was measured in numberof-turns when screwing the screw out or into the disc. Initially, the screw was screwed in firmly in the wooden disc. Thereafter, it was screwed out, a half turn each time, to increase the gap between wood and screw gradually until reaching 5 turns. Then it was screwed into the disc again with a half turn each time until reaching the tightscrew state again.

Finally, it was screwed out again exactly as before until reaching 5 turns. By this way, 10 different contact and stiffness grades (linearly distributed between 0.5 and 5 turns) were simulated three times and the corresponding power spectra were measured. Hence, the dataset available for this work consisted of 30 power spectra and the corresponding numbers of turns.

\subsection{Methodology}

1) Partial Least Squares (PLS): PLS is a multivariate

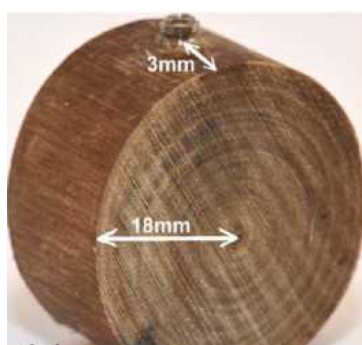

(a)

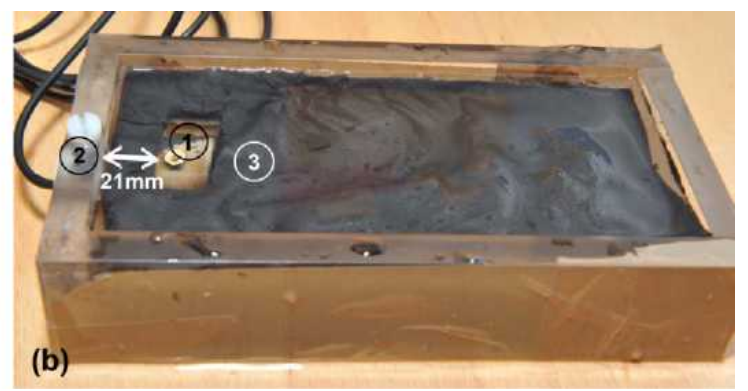

Figure 1. (a) Metal screw screwed into a wooden disc. (b) The experimental setup where the phantom, labeled with (1) and presented in Figure 1(a), is immersed into a pool filled with water and a soft-tissue equivalent material, labeled with (3). An ultrasonic transducer, labeled with (2), is mounted $21 \mathrm{~mm}$ away from the edge of the phantom. 
statistical framework which includes a wide class of methods and is used for processing, interpreting and analyzing data, measurements and observations in a wide range of fields and applications. An overview of PLS is presented by Rosipal and Kramer [12].

The pioneering work of introducing PLS was mainly done by Herman Wold in 1966 [13] and 1975 [14]. Since then, PLS has received great attention in many research fields. The basic principle of this method is to find and use a small number of uncorrelated variables (known as components or latent variables) to explain as much covariance as possible between the two blocks of explanatory $X$ and dependent $Y$ variables; where each column of the $X$ - and $Y$-matrices contains one variable.

At first, the $X$ - and $Y$-variables are preprocessed to make their distributions fairly symmetrical. The $n^{\text {th }}$ root transformation (where $\mathrm{n}$ is a real number) can be used to compress the dynamic range of these variables so that the result of dividing the mean value by the standard deviation (of this variable) will be around one. After that, a normalization technique called whitening [15] is performed to scale the data into zero-mean and unit-variance.

The general PLS model is described as follows:

$$
X=T P+E, Y=T Q^{T}+F
$$

where $X$ is an $n x m$ matrix of predictors, $Y$ is an $n x p$ matrix of responses, $T$ is an $n x l$ matrix of factors, $P$ and $Q$ are $m x l$ and $p x l$ loading matrices (of weight coefficients), respectively, and matrices $E$ and $F$ contain error terms.

There exist a number of PLS algorithms to estimate the factor and loading matrices $T, P$ and $Q$. Most of these algorithms estimate the linear regression between $X$ and $Y$ as:

$$
Y=X B+N
$$

where $Y$ contains $n$ cases and $m$ dependent variables, $X$ contains $n$ cases and $p$ independent variables, $B$ contains pxm regression coefficients (reflecting the covariance structure between $Y$ and $X$ ), and $N$ is a noise term of the same size as $Y$.

2) Using PLS analysis: There exist many different ways of performing PLS analysis. In this work the non-linear iterative partial least squares algorithm (NIPALS; [14]) is used. The first step of the NIPALS algorithm is to create two matrices $E=X$ and $F=Y$, where the columns of $X$ contain the measured ultrasonic power spectra (which are our independent variables), while $Y$ is a vector contains the corresponding dependent variables that are desired to be estimated.

The second step is to preprocess and normalize both $X$ and $Y$. Each element of vector $Y$ is transformed (by choosing an appropriate power value) so that the result of dividing its mean value by its standard deviation will be around one. After that, whitening is applied to $Y$. Matrix
$X$ is also whitened, but by employing two iterative normalisation approaches, where a number of alternating spectral-wise (denoted as Sw and performed row-wise in matrix $X$ ) and band-wise (denoted as Bw and performed column-wise in matrix $X$ ) whitening operations are performed, as described in [16]. When performing Sw-whitening, each spectrum (which corresponds to one row in matrix $X$ ) is whitened, while each column of $X$ (which corresponds to one spectral band) is whitened when Bwwhitening is performed. In the first iterative normalisation approach, a series of alternating Sw- and Bw-whitening operations, beginning and ending with Sw-whitening operations, were performed. On the other hand, the second iterative approach started with a Bw-whitening operation and ended with a Sw-whitening operation.

After that, the PLS algorithm starts and a series of iterative operations are performed until convergence of the result is achieved. At this point, the whole set of latent variables are calculated, as explained by Abdi [17].

In order to avoid over modeling, the number of latent variables to be included in the PLS model should be determined. A rule of thumb is that each latent variable used in the final model corresponds to five or six independent observations in the training dataset. (Rhiel et al. [18]).

By this way, an upper limit for how many latent variables to include has been defined. The remaining question is how many of these latent variables are enough to include. A popular way to know that is by calculating the relative error value which is zero when perfect prediction is achieved. Otherwise, it is always a positive number. The PLS model is improved as long as adding more latent variables lowers the relative error value. The optimal number of latent variables is found when the relative error value begins to increase [17].

3) Cross validation: To evaluate the usefulness and efficiency of the PLS model, cross validation is necessary. Leave-one-out cross validation is an efficient evaluation method when few observations are available. Only one pair of variables at a time is removed from the reference dataset and the excluded dependent parameter (which is desired to be estimated) is considered as unknown. The rest of the data samples are considered as a training dataset and the excluded measured spectrum is fed into the trained model to estimate the excluded dependent parameter.

The estimated parameter values are finally compared to the real values, to evaluate the performance of the PLS model. Both of the relative error and the coefficient of determination $\mathrm{R}^{2}$ can be used as evaluation measure.

\section{Experimental Results}

The gap between the jawbone and the dental implant may 
vary between zero and $0.1 \mathrm{~mm}$, which is the initial gap size when inserting a screw-type dental implant [19]. The gap-size variation corresponds to a variation of the contact and the stiffness grade between the implant and the jawbone. However, what is more interesting and important to know is if the osseointegration process is complete or not, and if this process is progressing towards obtaining a better intimate contact, between the bone and the implant, or not. An efficient way to simulate varying grades of osseointegration is to use the experimental setup and the phantom described previously.

The nonlinear propagation of the ultrasonic waves through the wooden disc, the metal screw and the water-filled gap between them will gradually deform the shape of the waves. Therefore, in addition to the effect of Fourier transforming the time-signal received by the transducer, higher harmonic frequencies (defined as integer multiples of the fundamental frequency of the ultrasonic transducer) appear in the power spectrum. The frequency of the peak of the first harmonic was shifted from $2 \mathrm{MHz}$ down to $1.92 \mathrm{MHz}$. The second harmonic peak was at around $3.84 \mathrm{MHz}$, the third one was at around $5.76 \mathrm{MHz}$ and so on, as shown in Figure 2. Low pass filtering was used to suppress the noise and get smoother spectra.

It is essential to ensure the repeatability of the experiments to validate the capability of an ultrasonic measurement system. Therefore, the experiments were repeated using different initial conditions and the measured power spectra were compared. The resulting standard deviations, when comparing spectra corresponding to the same contact level, were mainly limited to $1 \%$.

Figure 2 shows a comparison between two power spectra; one corresponds to a tight screw and the other one corresponds to a loose screw. It is possible to observe differences between these spectra, at the higher harmonics. These differences are automatically utilized by the PLS algorithm to be able to estimate the corresponding stiffness grade which is measured in number-of-turns when screwing the metal screw out or into the wooden disc. Before applying the PLS algorithm, the power spectra were preprocessed according to the guidelines mentioned previously.

Figure 3 presents a comparison between real and estimated stiffness grades (measured in number-of-turns), with a coefficient of determination $\mathrm{R}^{2}$ of $96.4 \%$ and $\mathrm{a}$ mean absolute error of \pm 0.23 turns.

\section{Discussion and Conclusions}

Although the obtained results indicate the usefulness and efficiency of the used approach, the transducer was attached to the phantom at approximately the same position, angle and direction during the whole experiment.

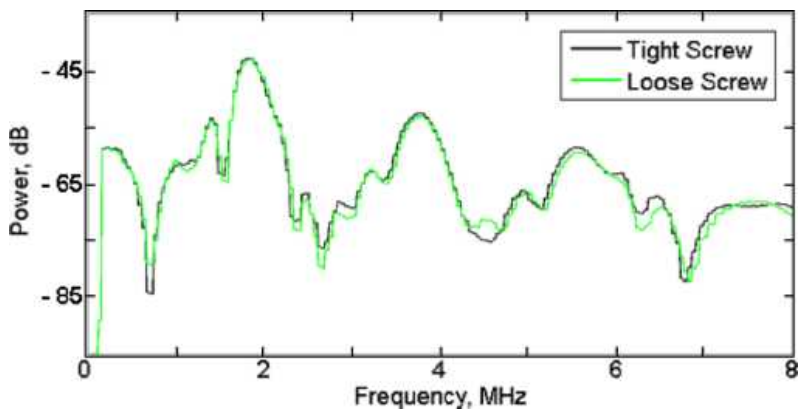

Figure 2. A comparison between two power spectra; a power spectrum of a tight screw and another one of a loose screw.

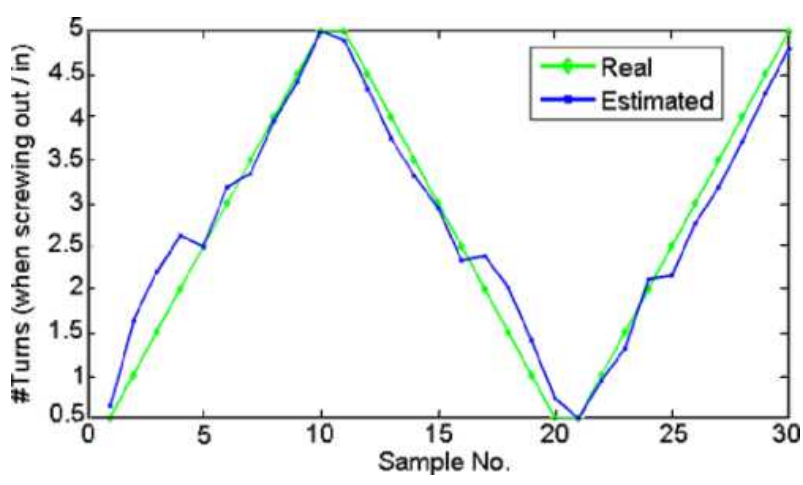

Figure 3. A comparison between real and estimated number-of-tums when screwing the screw out, then in, then out again. Various number-of-turns correspond to various stiffness grades between the screw and wood.

Minor changes occurred when trying to screw in or out the screw. The spectral measurements will change considerably when measuring at different parts of the phantom and from different directions or angles. However, it is possible to normalize these spectra and make them useable, since the method proposed and used in this work doesn't rely on comparing amplitudes of one or several peaks found at certain frequency intervals. The new method makes instead use of the shape of the whole spectrum which makes it more efficient and practical so that it can be used outside the laboratory without the need for controlled measuring conditions. Furthermore, it is not necessary to manually identify the most useful spectral regions where most variations among the spectra are visible. The significance of the new method is that it is objective, non-invasive, fast, accurate, automatic and reproducible.

\section{REFERENCES}

[1] P. I. Branemark, U. Breine, R. Adell, B. O. Hansson, J. Lindstrom and A. Ohlsson, "Intra-Osseous Anchorage of Dental Prostheses: I. Experimental Studies," Journal of Plastic and Reconstructive Surgery and Hand Surgery, Informa Healthcare, Vol. 3, No. 2, 1969, pp. 81-100. 
[2] D. W. Fitting and L. Adler, "Ultrasonic Spectral Analysis for Nondestructive Evaluation,” Plenum Press, New York, 1981. http://dx.doi.org/10.1007/978-1-4613-3126-1

[3] S. F. Lin, L. C. Pan, S. Y. Lee, Y. H. Peng and T. C. Hsiao, "Resonance Frequency Analysis for Osseointegration in Four Surgical Conditions of Dental Implants," Proceedings of the 23rd Annual International Conference of the IEEE Engineering in Medicine and Biology Society, Vol. 3, 2001, pp. 2998-3001.

[4] R. L. C. Pan and S. H. Ying, "Mechanical Properties of Bone-Implant Interface: An in Vitro Model for the Comparison of Stability Parameters Affecting Various Stages during Osseointegration for Dental Implant," IEMBS'04 26th Annual International Conference of the IEEE Engineering in Medicine and Biology Society, Vol. 2, 2005, pp. 5050-5052.

[5] P. Valderrama, T. W. Oates, A. A. Jones, J. Simpson, J. D. Schoolfield and D. L. Cochran, "Evaluation of Two Different Resonance Frequency Devices to Detect Implant Stability: A Clinical Trial,” Journal of Periodontology, American Academy of Periodontology, Vol. 78, No. 2, 2007, pp. 262-272. http://dx.doi.org/10.1902/jop.2007.060143

[6] V. Pattijn, S. V. N. Jaecques, E. De Smet, L. Muraru, C. Van Lierde, G. Van der Perre, I. Naert and J. V. Sloten, "Resonance Frequency Analysis of Implants in the Guinea Pig Model: Influence of Boundary Conditions and Orientation of the Transducer," Medical Engineering \& Physics, Vol. 29, No. 2, 2007, pp. 182-190. http://dx.doi.org/10.1016/j.medengphy.2006.02.010

[7] M. S. De Almeida, C. D. Maciel and J. C. Pereira, "Proposal for an Ultrasonic Tool to Monitor the Osseointegration of Dental Implants," Sensors, Molecular Diversity Preservation International, Vol. 7, No. 7, 2007, pp. 12241237.

[8] V. Mathieu, F. Anagnostou, E. Soffer and G. Haiat, “Ultrasonic Evaluation of Dental Implant Biomechanical Stability: An in Vitro Study," Ultrasound in Medicine \& Biology, Vol. 37, No. 2, 2011, pp. 262-270. http://dx.doi.org/10.1016/j.ultrasmedbio.2010.10.008

[9] A. Walker, “The Encyclopedia of Wood," Quatro Publishing, London 2005, p. 192.
[10] A. Tampieri, S. Sprio, A. Rufini, I. G. Lesci and N. Roveri, "From Wood to Bone: Multi-Step Process to Convert Hierarchical Structures into Biomimetic Hydroxyapatite Scaffolds for Bone Tissue Engineering,” Journal of Materials Chemistry, Vol. 19, No. 28, 2009, pp. 49734980. http://dx.doi.org/10.1039/b900333a

[11] E. L. Madsen, J. A. Zagzebski, R. A. Banjavie and R. E. Jutila, "Tissue Mimicking Materials for Ultrasound Phantoms,” Medical Physics, Vol. 5, 1978, p. 391. http://dx.doi.org/10.1118/1.594483

[12] R. Rosipal and N. Kramer, "Overview and Recent Advances in Partial Least Squares,” Subspace, Latent Structure and Feature Selection: Statistical and Optimization Perspectives Workshop, SLSFS 2005; Revised Selected Papers, Springer-Verlag Inc., New York, 2006, pp. 34-51. http://dx.doi.org/10.1007/11752790_2

[13] H. Wold, "Nonlinear Estimation by Iterative Least Squares Procedures,” In: F. N. David, Ed., Festschrift for J. Neyman, Wiley, New York, 1966, p. 411.

[14] H. Wold, "Path Models with Latent Variables: The NIPALS Approach,” Quantitative Sociology: International Perspectives on Mathematical and Statistical Modeling, 1975, pp. 307-357.

[15] Y. C. Eldar and A. V. Oppenheim, "MMSE Whitening and Subspace Whitening," IEEE Transactions on Information Theory, Vol. 49, No. 7, 2003, pp. 1846-1851. http://dx.doi.org/10.1109/TIT.2003.813507

[16] H. Hamid Muhammed, "Hyperspectral Crop Reflectance Data for Characterising and Estimating Fungal Disease Severity in Wheat,” Biosystems Engineering, Vol. 91, No. 1, 2005, pp. 9-20. http://dx.doi.org/10.1016/j.biosystemseng.2005.02.007

[17] H. Abdi, "Partial Least Square Regression,” Encyclopedia for Research Methods for the Social Sciences, 2003.

[18] M. Rhiel, M. B. Cohen, D. W. Murhammer and M. A. Arnold, "Nondestructive Near-Infrared Spectroscopic Measurement of Multiple Analytes in Undiluted Samples of Serum-Based Cell Culture Media,” University Of Iowa, 2001.

[19] R. K. Schenk and D. Buser, "Osseointegration: A Reality," Periodontology, Vol. 17, No. 1, 1998, pp. 22-35. 